\title{
A new method for the assessment of endothelial function with peripheral arterial volume
}

\author{
Daoyuan $\mathrm{Si}^{1} \mathbb{B}$, Lujia $\mathrm{Ni}^{2}$, Yunfei Wang ${ }^{1}$, Jinsha Liu', Jining Yang ${ }^{1}$ and Ping Yang ${ }^{1 *}$
}

\begin{abstract}
Background: Currently, many methodological approaches have been developed to assess peripheral endothelial function. However, a development of the noninvasive and automated technique for routinely assessing endothelial function is still required. We evaluated the potential value of a new method to measure peripheral endothelial function with reactive hyperemia peripheral arterial volume (RH-PAV) in patients with chest pain.

Methods: We used a novel oximeter-like probe to detect the peripheral arterial volume (PAV) of the finger and compared it with brachial flow-mediated dilation (FMD) performed in 93 consecutive patients with chest pain. The RH-PAV index was defined as the ratio of the digital pulse volume during reactive hyperemia relative to the baseline.

Results: Ninety-three patients (53 men, $58 \pm 5$ years) completed the study, and 53 patients demonstrated coronary artery disease (CAD) following scheduled coronary angiography. There was a moderate linear relationship between PAV and FMD $(r=0.69, p<0.01)$. Similar to FMD, PAV was more impaired in patients who have more cardiovascular risk factors (CRFs). The subjects with CAD had lower PAV and FMD, compared with those without CAD $(1.05 \pm 0.23$ VS. $1.41 \pm 0.37, p<0.01 ; 6.7 \% \pm 2.9 \%$ VS. $10.4 \% \pm 2.9 \%, p<0.01$, respectively), and the relationships between FMD and PAV were also significant in both CAD $(r=0.54, p<0.01)$ and non-CAD $(r=0.62, p<0.01)$ patients.

Conclusions: Endothelial function of digital artery assessed with the novel PAV method demonstrated a profile similar to that of brachial artery measured with FMD. The hyperemia PAV was decreased by factors which were considered to impair endothelial function, suggesting that PAV has the potential to be a novel method to study endothelial function.
\end{abstract}

Keywords: Noninvasive measurement, Endothelial function, Peripheral arterial volume, Flow mediated dilation, Photoplethysmography

\section{Background}

Cardiovascular diseases have become a global challenge in public health, and atherosclerosis is a major one that can lead to ischemia of the heart, brain or extremities, occasionally resulting in infarction [1]. Endothelial dysfunction is an integral part to each phase of atherosclerosis, from initiation to subsequent cardiovascular complications, but can be reversible at every stage,

\footnotetext{
* Correspondence: pyang@jlu.edu.cn

Daoyuan Si and Lujia Ni contributed equally to this manuscript and shared the first authorship.

'Department of Cardiology, Jilin Provincial Engineering Laboratory for Endothelial Function and Genetic Diagnosis of Cardiovascular Disease, China-Japan Union Hospital of Jilin University, Xiantai Street NO.126, Changchun, Jilin, China

Full list of author information is available at the end of the article
}

indicating early detection of endothelial dysfunction may have therapeutic and prognostic implications [2]. Therefore, the evaluation of the endothelial function is essential for investigating the pathophysiology of cardiovascular diseases, including coronary artery disease (CAD).

Endothelial function can be evaluated by detecting its capacity to perform the various functions, such as regulation of vasomotor tone which has become the most prevailing study endpoint $[3,4]$. The invasive assessment of endothelial function by catheterization was thought to be the "gold standard" in initial studies, but the invasive and costly nature prohibit its clinical utility [5]. Later on, flow mediated dilation (FMD), a noninvasive method of investigating peripheral artery, became widely used in

(c) The Author(s). 2018 Open Access This article is distributed under the terms of the Creative Commons Attribution 4.0 International License (http://creativecommons.org/licenses/by/4.0/), which permits unrestricted use, distribution, and 
the last few decades [6]. However, there were still several methodological challenges might limit its application in daily practice, such as being operator-dependent and systemic changes during testing [7]. Then peripheral arterial tonometry (PAT) gained increasing attention, based on similar physiological mechanisms as FMD, including the measurement of vasodilation in response to reactive hyperemia. Although growing evidence indicates that the promising technique potentially impacts the field of cardiovascular study, a number of methodological and clinical aspects still need to be clarified $[8,9]$.

Nonetheless, the development of a noninvasive and automated technique for routinely assessing endothelial function is still required, from a clinical perspective. The measurement of pulsatile volume changes of index finger during reactive hyperemia has been proved to be an effective way to evaluate endothelial function [10]. Forearm plethysmography was also shown to be a useful method to assess endothelial function [11, 12]. So we used a novel oximeter-like finger probe (peripheral arterial volume $[\mathrm{PAV}])$ to measure the changes of pulsatile blood flow volume during reactive hyperemia in our study, detecting the changes of hemoglobin $(\mathrm{Hb})$ flow. Our aim was to demonstrate the relationship between FMD and PAV in patients with chest pain.

\section{Methods}

\section{Study objective}

Patients were eligible for enrollment if they had chest pain, were scheduled to undergo coronary angiography (CAG) and were aged between 30 to 75 years. Exclusion criteria included acute coronary syndrome; left ventricular ejection fraction $<55 \%$; Raynaud's disease; atrial fibrillation; valvular heart disease; chronic respiratory; renal disease; thyroid disorders or sex hormone disorders. This study protocol conformed to the ethical guidelines of the Helsinki Declaration, approved by the ethical review board of China-Japan Union Hospital of Jilin University (2017040603), and the written informed consents were signed by all patients.

The following cardiovascular risk factors (CRFs) were evaluated in each individual: male sex; hypertension(systolic blood pressure $>140 \mathrm{mmHg}$ or/and diastolic blood pressure $>90 \mathrm{mmHg}$, or under antihypertensive treatment); diabetes mellitus (fasting glucose levels $>126 \mathrm{mg} / \mathrm{dL}$, or under antihyperglycemic treatment); hyperlipidemia (total serum cholesterol level $>200 \mathrm{mg} / \mathrm{dL}$ or taking lipidlowering treatment); family history of CAD; postmenopause; and current smoking(have smoked 100 cigarettes in their lifetime and currently smoke cigarettes).

In a dimly lit and temperature controlled room, PAV and FMD were performed on the early morning of the scheduled CAG. Participants were instructed to refrain from vasoactive medication, food, caffeine, tea, tobacco, or exercise for at least 12 hours before the measurement. CAD was defined as $50 \%$ or more luminal narrowing in one or more major epicardial vessels as determined by CAG.

\section{FMD testing}

FMD was measured according to the guidelines [13]. Following a 10-min equilibrium period, brachial artery diameter was assessed at baseline and post reactive hyperemia with a $12-\mathrm{MHz}$ linear-array peripheral vascular probe (Philips iE33 ultrasound system). The transient ischemia was induced by inflating a forearm blood pressure cuff to $50 \mathrm{mmHg}$ above patient's resting systolic blood pressure for $5 \mathrm{~min}$; then brachial artery diameter was measured at 1,2 and $3 \mathrm{~min}$ post cuff deflation. Percent change in flow-mediated dilation (\%FMD) was defined as the maximal brachial artery diameter due to reactive hyperemia compared to the baseline diameter. Percent change in nitroglycerin-mediated dilation (\%NMD) was defined as the maximal brachial artery diameter during a 3 min period following administration of nitroglycerin, compared with the baseline diameter. All measurements were performed twice by two independent observers who were blinded to the patients' information.

\section{PAV testing}

The basic principle of PAV is the same as FMD and PAT, this technique (Saintyear Medical Ltd., Shenzhen, China) includes a photo-plethysmographically based index finger probe to measure digital arterial volume changes accompanying pulse waves. Light-emitting diodes $(940 \mathrm{~nm})$ in conjunction with a light-sensitive sensor were used to assess the absorption of the infrared light. The calculation was made by the difference in digital artery hemoglobin flow between pre- and postocclusion of an upper limb.

The blood pressure cuff was placed on the left upper arm undergoing hyperemia testing, while the contralateral arm served as a control. PAV was performed by placing the probes on the index finger of each (Fig. 1). The occlusion was induced by inflating the cuff to $50 \mathrm{mmHg}$ above patient's resting systolic blood pressure for $5 \mathrm{~min}$. Reactive hyperemia PAV was defined as the ratio of the average pulse wave amplitude (PWA) during the 40-s period beginning after exactly $40 \mathrm{~s}$ of reactive hyperemia compared with the average PWA during the 40-s period beginning after exactly $40 \mathrm{~s}$ of pre-occlusion baseline. The result of PAV was calculated automatically normalizing to the contralateral arm to correct for any systemic changes during the test. The nitroglycerin response (PAV nitroglycerin) was calculated as the ratio of the average PWA during the 40-s period beginning exactly after $3 \mathrm{~min}$ after administration of nitroglycerin 
A

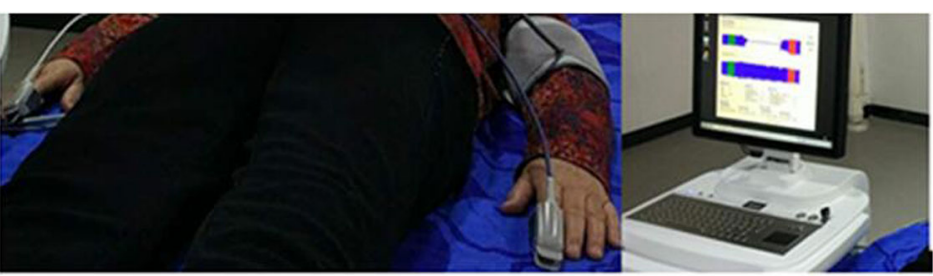

B

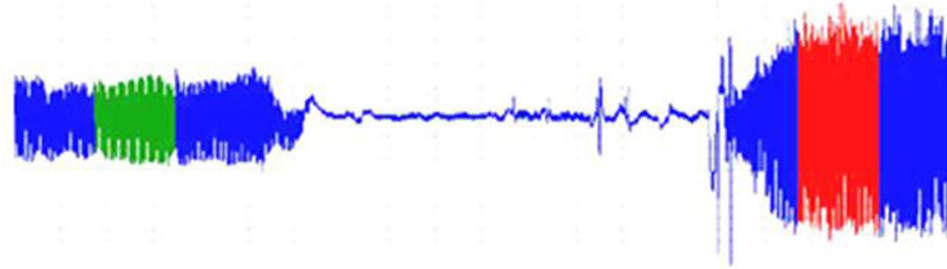

$\mathrm{C}$

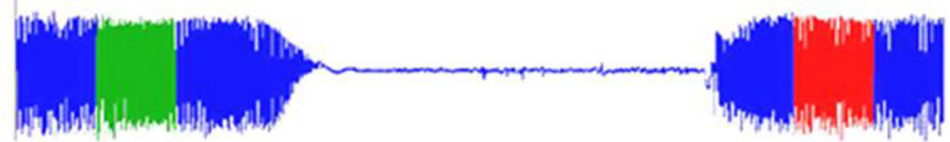

$\mathrm{D}$

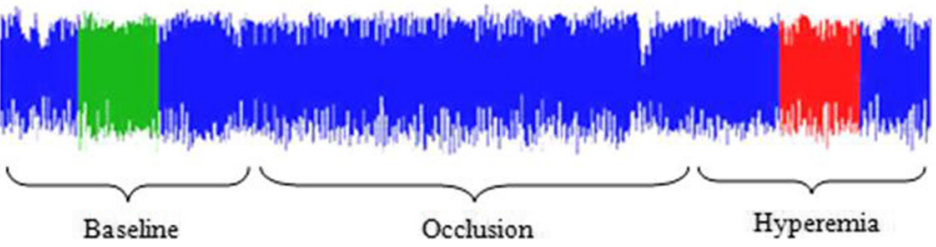

Fig. 1 a setup of PAV testing, $\mathbf{b}$ and $\mathbf{c}$, from the side undergoing hyperemia. $\mathbf{d}$ from the contralateral side. $\mathbf{b}$ Individual with non-CAD and fewer CRFs showing an increased PAV signal during hyperemia. C, Individual with CAD and more CRFs showing a blunted PAV response during hyperemia. D, PAV recording from the same patient with CAD and more CRFs in contralateral side

compared with the average PWA during the 40-s period beginning after exactly $40 \mathrm{~s}$ of the second baseline, and no contralateral correction was applied.

\section{Statistical analysis}

Data are presented as mean \pm standard deviation(SD). The two-sided t-test, Pearson rank correlation, and linear regression analysis were used to assess the relationship between FMD and PAV. Univariate analysis was used to evaluate the association between the PAV and various CRFs. Receiver operating characteristic (ROS) curves of FMD and PAV was performed to assess the discrimination performance of CAD. All analyses were done by SPSS 17 . 0 statistical software (SPSS Inc., Chicago, Illinois). $P \leq 0.05$ was considered statistically significant.

\section{Results}

\section{Study population}

Ninety-three individuals (58 \pm 5 years) completed the study, and 53 of them had CAD. We describe the characteristics of our study population through Table 1. Left ventricular ejection fraction $(62 \% \pm 2 \%)$ was normal in each patient, and approximately half of the patients had hypertension. $23 \%$ of subjects were reported a family history of CAD, $24 \%$ of subjects had diabetes mellitus, and $70 \%$ of subjects had hyperlipidemia.

\section{Relation between FMD and PAV}

The average of FMD and PAV were $8.3 \% \pm 3.4 \%$ (range $2.0-15.1 \%$ ) and $1.21 \pm 0.35$ (range $0.60-2.22$ ), respectively. Linear regression analysis revealed that FMD and PAV were significantly correlated in all patients

Table 1 Study population characteristics

\begin{tabular}{ll}
\hline Variable & $n=93$ \\
\hline Age(years) & $58 \pm 5$ \\
Male, $\mathrm{n}(\%)$ & $53(57)$ \\
Body mass index $\left(\mathrm{kg} / \mathrm{m}^{2}\right)$ & $24.6 \pm 3.3$ \\
Left ventricular ejection fraction(\%) & $62 \pm 3$ \\
Coronary artery disease & $53(57)$ \\
Systemic hypertension(\%) & $59(53)$ \\
Diabetes mellitus(\%) & $22(24)$ \\
Hyperlipidemia(\%) & $65(70)$ \\
Current smoking(\%) & $54(58)$ \\
Family history of coronary disease(\%) & $21(23)$ \\
Postmenopause(\% of women) & $30(75)$ \\
\hline
\end{tabular}

Values are presented as mean \pm SD or number (\%) 
$(r=0.69, p<0.01)$ (Fig. 2a). The significant relationships were also identified between FMD and PAV in both CAD $(r=0.54, p<0.01)$ and non-CAD $(r=0.62$, $p<0.01$ ) populations (Fig. $2 \mathrm{~b}$ ). The average of NMD was $13.9 \% \pm 4.3 \%$ (range $5.1-26 \%$ ), whereas the average of nitroglycerin-PAV was $1.52 \pm 0.37$ (range 0.81-2.7, $r=0.65$, $p<0.01)$.

\section{Relation between CRFs and PAV}

The average number of CRFs was $3.0 \pm 1.2$ in each patient. Spearman correlation analysis revealed a significant relationship between the number of CRFs and PAV $(r=0.45$, $p<0.01)$, as well as FMD $(r=0.53, p<0.01)$, and the trend is similar (Fig. 3). Eight patients with $<2$ CRFs had the PAV of $1.65 \pm 0.36,51$ patients with 2 to 3 CRFs had the PAV of $1.25 \pm 0.35$, and 34 patients with $>3$ CRFs had the PAV of $1.04 \pm 0.21$ ( $<2$ vs. $2-3$ CRFs $P<0.05,2$-3 vs. $>3$ CRFs $P<0.01,<2$ vs. $>3$ CRFs $P<0.01)$. The PAV was significantly lower in patients with hypertension $(1.10 \pm 0.31)$, hyperlipidemia $(1.11 \pm 0.31)$, diabetes mellitus $(1.02 \pm 0.23)$ or family history of $\mathrm{CAD}(1.17 \pm 0.25)$ than those without such $\operatorname{CRF}(1.32 \pm 0.35 P<0.01,1.30 \pm 0.35 P<0.01$, $1.26 \pm 0.36 P<0.01,1.22 \pm 0.37$, respectively). Postmenopausal women $(1.13 \pm 0.34)$ had lower PAV than premenopausal ones $(1.5 \pm 0.32 \quad P<0.01)$. There was no significant relationship between the PAV and the gender, age, or current smoking.

\section{Relation between CAD and PAV}

The subjects with CAD had lower PAV $(1.05 \pm 0.23)$ and FMD $(6.7 \pm 2.9 \%)$ compared with those without CAD $(1.41 \pm 0.37,10.4 \pm 2.9 \%, P<0.01$ for both). The area under the curve (AUC) of PAV and FMD was 0.785 (95\%CI 0.694-0.877, $P<0.01$ ) and 0.810 (95\%CI $0.725-0$. $895, P<0.01)$ for the prediction of CAD in ROC curves (Fig. 4). There was no significant difference between the AUCs of PAV and FMD $(\mathrm{Z}=0.54<1.96, P>0.05)$ with the pairwise comparison of ROC curve test. The sensitivity and specificity of PAV were 83 and $65 \%$, while those of
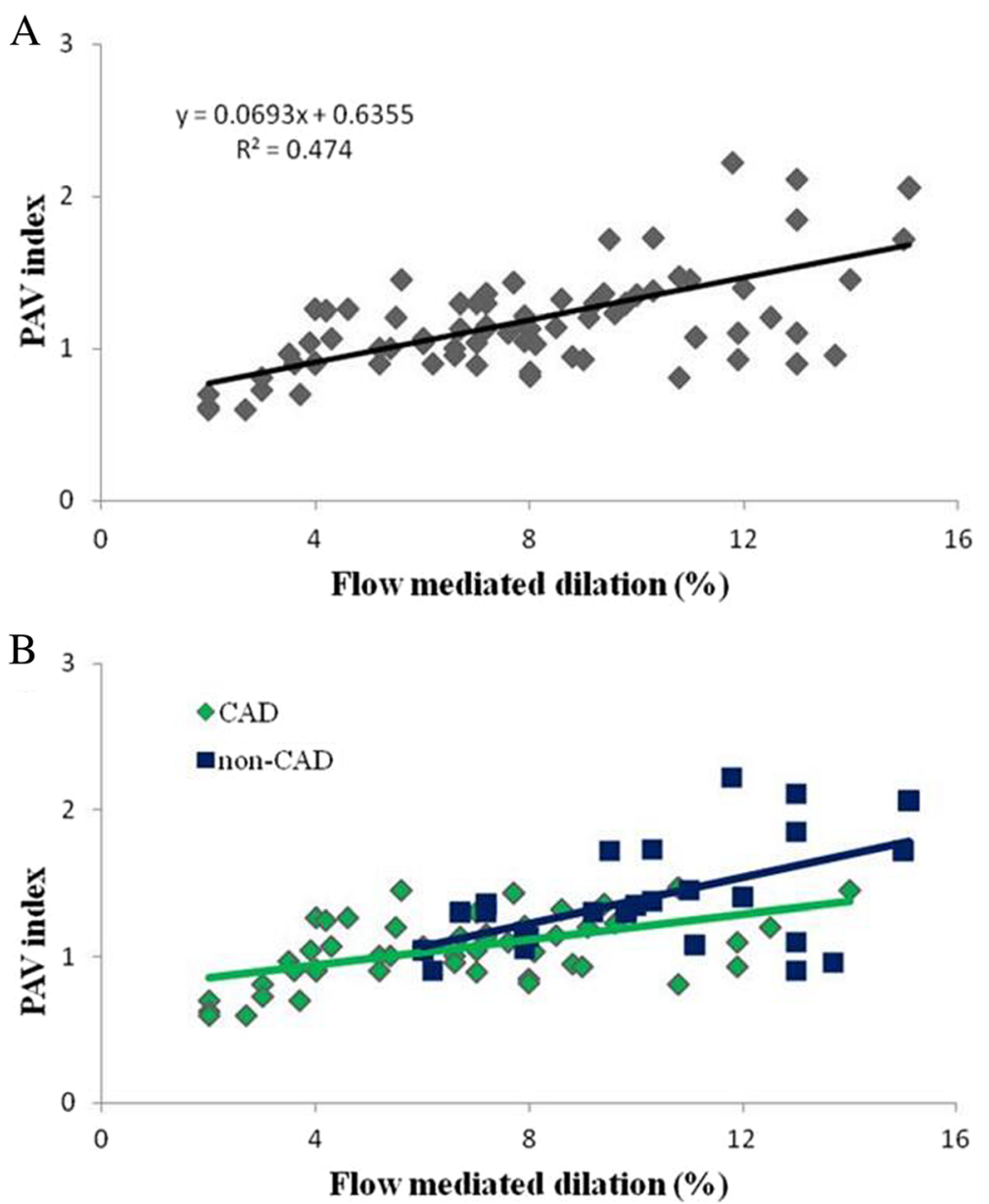

Fig. 2 a Linear regression evaluation of the relation between PAV index and FMD of the brachial artery in all patients $(r=0.69, P<0.01)$. $\mathbf{b}$ Linear regression evaluation of relation between PAV index and FMD in CAD $(r=0.54, P<0.01, n=53)$ and non-CAD $(r=0.62, P<0.01, n=40)$ population 

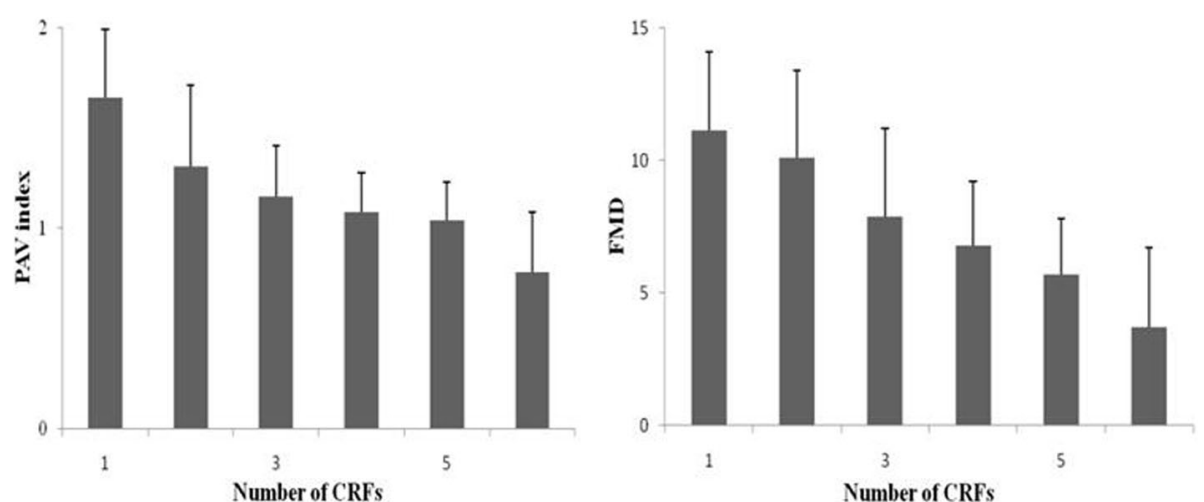

Fig. 3 Similar to brachial artery FMD, the PAV index generally correlated with the numbers of CRFs $(r=0.53, P<0.01$ for FMD, $r=0.45, P<0.01$ for PAV). Values are expressed as the mean \pm SD

FMD were 85 and $63 \%$ for detection of CAD when 1.28 of PAV and $9.8 \%$ of FMD was identified as the cutoff points.

\section{Discussion}

Our pilot study was performed to evaluate the feasibility of reactive hyperemia PAV to assess endothelial function of the peripheral artery in patients with chest pain. The data demonstrated that the significant relationship between PAV hyperemia ratio and brachial artery FMD not only existed in all subjects but also in both CAD and non-CAD groups. Similar to FMD, peripheral arterial reactivity, defined as the PAV, also correlated with traditional CRFs and CAD. Since there is a significant relationship between abnormalities in the central and peripheral arteries in the process of atherosclerosis, peripheral vascular endothelial function testing has been

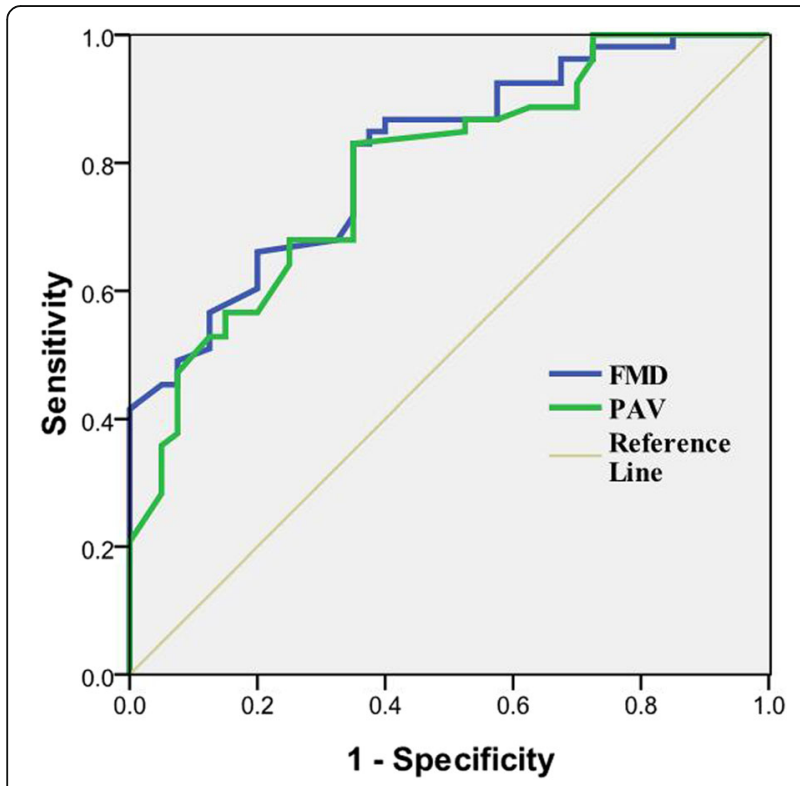

Fig. 4 ROCS of FMD and PAV for the prediction of coronary heart disease evolving to be more feasible for daily test and screening large numbers of people [2]. Our findings were consistent with prior studies of peripheral vascular endothelial function testing $[14,15]$. The PAV technique could represent a new method to assess endothelial function from peripheral vascular beds, contributing to the development of noninvasive measurement of endothelial function. Although the brachial and digital measurements of vascular function may reveal distinct information and reflect different characterizations of a disease stage, the association of both was observed in aged patients or who have more advanced CRFs in prior studies $[16,17]$.

The PAV was lower in patients with diabetes mellitus, hypertension, hyperlipidemia, or family history of CAD, but there was no significant relationship between the decreased PAV and age, gender or current smoking. Previous studies have reported that the prevalence of reduced FMD increased substantially with advancing age, and the association attributed to alterations in the production of nitric oxide and oxidant species $[17,18]$. In contrast, low PAV showed little variability with advancing age in our study. Actually increasing age also did not associate with abnormal PAT in several studies [16, 19]. The findings may potentially be explained by the counterbalance physiologic changes in the finger microvessels with aging that tend to preserve the reactive hyperemia $[15,16]$. Recently Ulrike et al. [20] determined that PAT ratio increased significantly with increasing age in 445 children and adolescents, so it was also possible because of a large number of old patients who were enrolled in our study. Men always had lower values for noninvasive measurements of endothelial function, such as FMD, in prior reports $[15,17,21,22]$, but sex was not found to affect PAV ratio in our study. The possible explanation might be the high rate of postmenopausal women (75\%) enrolled in the present study. The enrollment bias might be the reason why current smoking had no significant effect on PAV in the study. 
Our study demonstrated that patients with CAD had attenuated PAV, compared to those without CAD, and the discrimination value of CAD existence was similar between PAV and FMD. Although it has been reported that peripheral vascular endothelial function measured by blood flow or reactive hyperemia after cuff release are not solely nitro oxygen-mediated, it possesses independent predictive value [16]. Vasodilation measurements of conduit artery and microarray probably reflect a distinct aspect of vascular function complementarily $[2,16]$. Our findings consisted of previous studies about other measurements of endothelial function including FMD and PAT, suggesting that assessment of peripheral endothelial function using PAV could be potentially valuable for identifying patients with CAD [14, 23, 24].

\section{PAV technique}

The absence of major muscle, high vascular density and good perfusion of the finger make it accessible for measuring changes in the volume of blood flow [25]. The photoplethysmographic recordings of PAV were derived from the index finger in the study, and the similar technique was also used in the analysis of digital volume pulse (DVP) and pulse oximetry [26, 27]. Technically speaking, PAV is likely to be the surrogate for arterial distensibility of the vascular digital district, as the blood volume in the veins and capillaries, as well as the bone, fat, and skin, remain relatively constant while the blood volume in the artery increases during systole and decreases during diastole. According to the Beer-Lambert Law in a modeled blood vessel, simply measuring the absolute absorbance in the finger would be an inaccurate estimate of arterial volume since distended distal venous blood volume would also contribute to the value measured. Nevertheless, the PAV system is able to use similar mathematics which is also used in the pulse oximeter to extract only arterial absorbance of $\mathrm{Hb}$ from the total signal by measuring changes in absorbance over time [26]. The influence of these relatively stationary facts, such as distended distal venous caused by occlusion, can be excluded from the automatical calculation of PAV. So instead of thimbleshaped pneumatic finger probes, used in PAT test and be considered vulnerable and disposable [28], reusable finger probes are used in PAV technique. That may potentially contribute to the daily use in the future.

\section{Limitations and future directions}

As described above, the PAV technique is operator independent, analyzing automatically, requiring less training and the contralateral arm serves as its internal control that can be used to correct for any systemic changes during the test. Several limitations of this study and the technique deserve to be mentioned. First, only traditional CRFs were considered and other risk factors may also have potential to impair endothelial function. Gender, age and current smoking were acknowledged to impair endothelial function, but the relationships between them and PAV were not significant, the bias may affect the result. Second, reproducibility is important when vascular reactivity tests are compared [29]. Future studies should investigate the variation of PAV and explore the pathophysiology of PAV, including the anatomical and hemodynamic determinants. Third, Due to the different absorbance of oxygenated and deoxygenated hemoglobin in $940 \mathrm{~nm}$ wavelength infrared light, the calculation of PAV that takes total hemoglobin into account may be inaccurate in patients with low oxygen saturation. So chronic respiratory disease was included in the exclusion criteria of our study. $800 \mathrm{~nm}$ wavelength probably should be studied, where the absorbance of oxygenated and deoxygenated hemoglobin are equal, as well as an improvement strategy. Finally, the population enrolled was relatively small and all subjects underwent coronary angiography for clinically suspected CAD. Therefore, selection bias may affect the results, larger cohorts are needed for further study.

\section{Conclusion}

Our data indicated that the novel PAV method showed similar patterns of abnormality to FMD in patients with chest pain. PAV is influenced by factors proven to affect endothelial function, such as the presence of CRF and CAD. In addition, PAV is potentially useful for identifying patients at high risk for CAD, suggesting that PAV has the potential to be a novel noninvasive method in the field of cardiovascular research.

\begin{abstract}
Abbreviations
AUC: Area under curve; CAD: Coronary artery disease; CAG: Coronary angiography; CRF: Cardiovascular risk factor; FMD: Flow mediated dilation; Hb: Hemoglobin; NMD: Nitroglycerin mediated dilation; PAT: Peripheral arterial tonometry; PAV: Peripheral arterial volume; PWA: Pulse wave amplitude; ROC: Receiver operating characteristic curve; SD: Standard deviation
\end{abstract}

\section{Funding}

This work was supported by the grants from Excellent Youth Foundation of Science and Technology of Jilin Province (No.20180520054JH) and Project of Development and Reform Commission of Jilin Province (No. 2016C026).

\section{Availability of data and materials \\ The datasets used and/or analyzed during the current study are available from the corresponding author on reasonable request.}

\section{Authors' contributions}

DS designed the study, performed statistical analysis, and was a major contributor in writing the manuscript. LN contributed to conception, design, and data acquisition. $Y W, J L$, and JY were involved in drafting the manuscript. PY made substantial contributions to conception of the study and interpretation of data and revised the manuscript. All authors read and approved the final manuscript.

Ethics approval and consent to participate

This study was approved by the ethical review board of China-Japan Union Hospital of Jilin University (2017040603). All individuals signed the written 
informed consents to the use of their clinical data for the purpose of research in the study.

\section{Competing interests}

Author Ping Yang has been a consultant for Saintyear Medical Ltd., which owns patent rights to PAV technique. Other authors have nothing to disclose.

\section{Publisher's Note}

Springer Nature remains neutral with regard to jurisdictional claims in published maps and institutional affiliations.

\section{Author details}

'Department of Cardiology, Jilin Provincial Engineering Laboratory for Endothelial Function and Genetic Diagnosis of Cardiovascular Disease, China-Japan Union Hospital of Jilin University, Xiantai Street NO.126, Changchun, Jilin, China. ${ }^{2}$ Department of Ultrasonography, China-Japan Union Hospital of Jilin University, Changchun, Jilin, China.

Received: 1 January 2018 Accepted: 26 April 2018

\section{Published online: 04 May 2018}

\section{References}

1. Mozaffarian D, Benjamin EJ, Go AS, Arnett DK, Blaha MJ, Cushman M, Das SR, de Ferranti S, Despres JP, Fullerton HJ, et al. Heart disease and stroke Statistics-2016 update: a report from the American Heart Association. Circulation. 2016;133(4):e38-360.

2. Matsuzawa Y, Lerman A. Endothelial dysfunction and coronary artery disease: assessment, prognosis, and treatment. Coron Artery Dis. 2014;25(8): 713-24

3. Higashi Y. Assessment of endothelial function. History, methodological aspects, and clinical perspectives. Int Heart J. 2015;56(2):125-34.

4. Mozos I, Malainer C, Horbanczuk J, Gug C, Stoian D, Luca CT, Atanasov AG Inflammatory markers for arterial stiffness in cardiovascular diseases. Front Immunol. 2017;8:1058.

5. Layland J, Nerlekar N, Palmer S, Berry C, Oldroyd K. Invasive assessment of the coronary microcirculation in the catheter laboratory. Int J Cardiol. 2015; 199:141-9.

6. Celermajer DS, Sorensen KE, Gooch VM, Spiegelhalter DJ, Miller OI, Sullivan ID, Lloyd JK, Deanfield JE. Non-invasive detection of endothelial dysfunction in children and adults at risk of atherosclerosis. Lancet. 1992;340(8828): $1111-5$.

7. Thijssen DH, Black MA, Pyke KE, Padilla J, Atkinson G, Harris RA, Parker B, Widlansky ME, Tschakovsky ME, Green DJ. Assessment of flow-mediated dilation in humans: a methodological and physiological guideline. Am J Physiol Heart Circ Physiol. 2011;300(1):H2-12.

8. Frolow M, Drozdz A, Kowalewska A, Nizankowski R, Chlopicki S Comprehensive assessment of vascular health in patients; towards endothelium-guided therapy. Pharmacol Rep. 2015:67(4):786-92.

9. Hedetoft M, Olsen NV. Evaluation of endothelial function by peripheral arterial tonometry and relation with the nitric oxide pathway. Nitric Oxide. 2014;42:1-8.

10. Brant LC, Wang N, Ojeda FM, LaValley M, Barreto SM, Benjamin EJ, Mitchell GF, Vasan RS, Palmisano JN, Munzel T, et al. Relations of metabolically healthy and unhealthy obesity to digital vascular function in three community-based cohorts: a meta-analysis. J Am Heart Assoc. 2017;6(3): e004199.

11. Wilkinson IB, Webb DJ. Venous occlusion plethysmography in cardiovascular research: methodology and clinical applications. Br J Clin Pharmacol. 2001; 52(6):631-46

12. Tsuchiya K, Kirima K, Yoshizumi M, Tamaki T. New methods to evaluate endothelial function: evaluation of endothelial function by hemoglobinnitric oxide complex using electron paramagnetic resonance spectroscopy. J Pharmacol Sci. 2003;93(4):417-22.

13. Corretti MC, Anderson TJ, Benjamin EJ, Celermajer D, Charbonneau F, Creager MA, Deanfield J, Drexler H, Gerhard-Herman M, Herrington D, et al. Guidelines for the ultrasound assessment of endothelial-dependent flowmediated vasodilation of the brachial artery: a report of the international brachial artery reactivity task force. J Am Coll Cardiol. 2002;39(2):257-65.

14. Kuvin JT, Mammen A, Mooney P, Alsheikh-Ali AA, Karas RH. Assessment of peripheral vascular endothelial function in the ambulatory setting. Vasc Med. 2007;12(1):13-6.
15. Kuvin JT, Patel AR, Sliney KA, Pandian NG, Sheffy J, Schnall RP, Karas RH, Udelson JE. Assessment of peripheral vascular endothelial function with finger arterial pulse wave amplitude. Am Heart J. 2003;146(1):168-74.

16. Hamburg NM, Palmisano J, Larson MG, Sullivan LM, Lehman BT, Vasan RS, Levy D, Mitchell GF, Vita JA, Benjamin EJ. Relation of brachial and digital measures of vascular function in the community: the Framingham heart study. Hypertension. 2011;57(3):390-6.

17. Benjamin EJ, Larson MG, Keyes MJ, Mitchell GF, Vasan RS, Keaney JF Jr, Lehman BT, Fan S, Osypiuk E, Vita JA. Clinical correlates and heritability of flow-mediated dilation in the community: the Framingham heart study. Circulation. 2004;109(5):613-9.

18. Brandes RP, Fleming I, Busse R. Endothelial aging. Cardiovasc Res. 2005;66(2): 286-94.

19. Onder NS, Akpinar ME, Yigit O, Gor AP. Watch peripheral arterial tonometry in the diagnosis of obstructive sleep apnea: influence of aging. Laryngoscope. 2012;122(6):1409-14.

20. Mueller UM, Walther C, Adam J, Fikenzer K, Erbs S, Mende M, Adams V, Linke A, Schuler G. Endothelial function in children and adolescents is mainly influenced by age, sex and physical activity- an analysis of reactive hyperemic peripheral artery tonometry. Circ J. 2017;81(5):717-25.

21. Hamburg NM, Keyes MJ, Larson MG, Vasan RS, Schnabel R, Pryde MM, Mitchell GF, Sheffy J, Vita JA, Benjamin EJ. Cross-sectional relations of digital vascular function to cardiovascular risk factors in the Framingham heart study. Circulation. 2008;117(19):2467-74.

22. Yoo JK, Pinto MM, Kim HK, Hwang CL, Lim J, Handberg EM, Christou DD. Sex impacts the flow-mediated dilation response to acute aerobic exercise in older adults. Exp Gerontol. 2017;91:57-63.

23. Woo JS, Jang WS, Kim HS, Lee JH, Choi EY, Kim JB, Kim WS, Kim KS, Kim W. Comparison of peripheral arterial tonometry and flow-mediated vasodilation for assessment of the severity and complexity of coronary artery disease. Coron Artery Dis. 2014;25(5):421-6.

24. Mannheimer PD. The light-tissue interaction of pulse oximetry. Anesth Analg. 2007;105(6 Suppl):S10-7.

25. Chowienczyk PJ, Kelly RP, MacCallum H, Millasseau SC, Andersson TL, Gosling RG, Ritter JM, Anggard EE. Photoplethysmographic assessment of pulse wave reflection: blunted response to endothelium-dependent beta2adrenergic vasodilation in type II diabetes mellitus. J Am Coll Cardiol. 1999; 34(7):2007-14.

26. Chan ED, Chan MM. Pulse oximetry: understanding its basic principles facilitates appreciation of its limitations. Respir Med. 2013;107(6):789-99.

27. Alaei-Shahmiri F, Zhao Y, Sherriff J. Assessment of vascular function in individuals with hyperglycemia: a cross-sectional study of glucose - induced changes in digital volume pulse. J Diabetes Metab Disord. 2015;14:23.

28. Kandhai-Ragunath JJ, Jorstad HT, de Man FH, Peters RJ, von Birgelen C. Approaches for non-invasive assessment of endothelial function: focus on peripheral arterial tonometry. Neth Heart J. 2013;21(5):214-8.

29. Bruno RM, Gori T, Ghiadoni L. Endothelial function testing and cardiovascular disease: focus on peripheral arterial tonometry. Vasc Health Risk Manag. 2014;10:577-84.

\section{Ready to submit your research? Choose BMC and benefit from:}

- fast, convenient online submission

- thorough peer review by experienced researchers in your field

- rapid publication on acceptance

- support for research data, including large and complex data types

- gold Open Access which fosters wider collaboration and increased citations

- maximum visibility for your research: over $100 \mathrm{M}$ website views per year

At BMC, research is always in progress.

Learn more biomedcentral.com/submissions 